\title{
Endoscopic resection versus esophagectomy for early esophageal cancer: a meta-analysis
}

\author{
Hao Zheng ${ }^{\# \wedge}$, Ningning Kang ${ }^{\#}$, Yunlong Huang, Yuan Zhao, Renquan Zhang \\ Department of Thoracic Surgery, The First Affiliated Hospital of Anhui Medical University, Hefei, China \\ Contributions: (I) Conception and design: H Zheng; (II) Administrative support: R Zhang; (III) Provision of study materials or patients: H Zheng, N \\ Kang; (IV) Collection and assembly of data: H Zheng, Y Huang; (V) Data analysis and interpretation: H Zheng, N Kang, Y Zhao; (VI) Manuscript \\ writing: All authors; (VII) Final approval of manuscript: All authors. \\ "These authors contributed equally to this work. \\ Correspondence to: Dr. Renquan Zhang, MD. Department of Thoracic Surgery, The First Affiliated Hospital of Anhui Medical University, Hefei \\ 230000, China. Email: zhangrenquan@live.cn.
}

Background: Esophagectomy is the standard treatment for early-stage esophageal cancer but is associated with high morbidity and mortality. Thus, endoscopic resection is increasingly used as an alternative option. However, the literature is inconsistent regarding the efficacy of these treatments. This meta-analysis aimed to compare the efficacy and safety of these two treatments.

Methods: A systematic electronic search of PubMed, EMBASE, and the Cochrane Library was performed for studies comparing endoscopic resection and surgery for early-stage esophageal cancer. The overall survival, tumor recurrence, major adverse events, procedure-related mortality, and R0 resection rates were investigated. Forest plots were constructed based on the random-effects model.

Results: We found 15 studies involving 2,467 and 2,264 patients who underwent endoscopic resection and surgery, respectively. The meta-analysis showed that patients undergoing endoscopic resection had significantly fewer major adverse events (relative risk, 0.46; 95\% confidence interval, 0.33-0.64) and a lower procedure-related mortality rate (relative risk, 0.27 ; 95\% confidence interval, $0.10-0.73$ ) than those undergoing surgery. The number of postprocedural stricture events did not significantly differ between the two treatments (relative risk, 0.89; 95\% confidence interval, 0.53-1.49). Endoscopic resection was associated with higher recurrence rates (relative risk, 1.69; 95\% confidence interval, 0.99-2.89) and lower R0 resection rates (relative risk, $0.92 ; 95 \%$ confidence interval, 0.86-0.98) than surgery. There may be some advantage conferred by esophagectomy in the long-term survival outcomes (hazard ratio, 1.21; 95\% confidence interval, 1.02-1.43).

Discussion: Endoscopic resection is a minimally invasive and safe treatment for early-stage esophageal cancer. However, esophagectomy may be associated with better long-term survival.

Keywords: Endoscopic resection; esophagectomy; early esophageal cancer (EEC)

Submitted Jan 27, 2021. Accepted for publication May 14, 2021.

doi: $10.21037 /$ tcr-21-182

View this article at: https://dx.doi.org/10.21037/tcr-21-182

\section{Introduction}

Early esophageal cancer (EEC), defined as tumors are limited to the basement membrane (Tis), mucosa (T1a), or submucosal layer (T1b) of the esophageal wall, constitutes approximately $20 \%$ of all cases of esophageal cancer (1). Although the prognosis of locally advanced esophageal

\footnotetext{
$\wedge$ ORCID: 0000-0002-5552-1087.
} 
cancer is poor, the early detection and treatment will be associated with a much better outcome for patients with early disease. The reported 5 -year survival rate is $>90 \%$ in post-surgical T1a patients (2).

Esophagectomy plus lymph node dissection is generally associated with significant postoperative morbidity and mortality (3), and poor long-term quality of life (4), even in high volume and centers with multi-disciplinary care (5). Therefore, endoscopic resection (ER) might be an alternative option for the treatment of EEC as it is minimally invasive, promotes rapid recovery, and is relatively low-cost. However, ER is limited in that it is not accompanied by lymph node dissection. Although the risk of lymph node metastasis is very low when the tumor is limited to the mucosa, it can rise to $20 \%$ in cases of tumors involving the submucosa (6). This issue has challenged the effectiveness of ER as an alternative pathway to esophagectomy for EEC.

While several studies have evaluated the efficacy of ER and surgery for EEC, the results were inconsistent. Unfortunately, due to the high frequency of adverse events in those undergoing esophagectomy, it would be difficult to perform a randomized controlled trial to determine which of these two treatments is safer and more effective. To our knowledge, no meta-analysis has been conducted for this purpose. Therefore, we performed a meta-analysis to further evaluate the efficacy and safety of ER and esophagectomy in the treatment of EEC. We present the following article in accordance with the PRISMA reporting checklist (available at https://dx.doi. org/10.21037/tcr-21-182).

\section{Methods}

\section{Study identification and selection criteria}

A systematic literature search was conducted to identify relevant studies that compared ER and surgery for EEC. PubMed, EMBASE, and the Cochrane Library were independently searched by two authors (H Zheng and N Kang). The following search terms were used: ("endoscopic mucosal resection" OR "EMR" OR "endoscopic submucosal dissection" OR "ESD" OR "endoscopic resection" OR "ER") AND (surgery OR operation) AND (early esophageal cancer). The literature search had no language restriction and ended on August 13, 2020. This meta-analysis was registered in PROSPERO (CRD42020181615).

The inclusion criteria were as follows: randomized controlled trials and non-randomized prospective and retrospective studies, analyses of both ER (endoscopic mucosal resection, endoscopic submucosal dissection (ESD), or both) and surgery for the treatment of EEC, and patients histologically diagnosed with EEC. The exclusion criteria were as follows: letters, case reports, comments, reviews, and studies with no full text or incomplete data available for analysis. If duplicate and multiple articles from the same team were retrieved, the most recent and informative publication was included in the meta-analysis.

\section{Data extraction}

All included studies and extracted the relevant data were assessed by two authors (H Zheng and $\mathrm{N}$ Kang), and a third author (Y Huang) decided the eligibility of any studies that these two authors did not agree upon. The following information was extracted: first author; year of publication; number, age, and sex of patients; follow-up duration; and outcome measures such as recurrence rate, $\mathrm{R} 0$ resection rate, procedure-related mortality, adverse event rates, postprocedural stricture events, and overall survival (OS) rates.

\section{Quality assessment}

The quality of the included studies was assessed by using the Newcastle-Ottawa scale, with some modifications to match the needs of this meta-analysis (7). Quality assessment was independently carried out by two authors ( $\mathrm{H}$ Zheng and $\mathrm{N}$ Kang). The quality of the studies was evaluated via the examination of four items: representativeness of the exposed cohort, comparability of the study groups, adequacy of follow up, and assessment of outcome. A quality score was awarded to each study, with a maximum of 10 points indicating the highest possible score. Studies achieving $\geq 5$ points were considered high quality.

\section{Statistical analysis}

We combined extracted data using R 3.6.1 software (version 3.6.1; https://www.r-project.org/). The hazard ratios (HRs) were extracted by using previously reported methods (8); one to obtain the HR value from the original paper, and another to estimate the HR value from related data. The estimated heterogeneity of the included researches were based on $\mathrm{I}^{2}$ test, with levels of heterogeneity defined as minor $\left(I^{2}=0-30 \%\right)$, moderate $\left(I^{2}=30-50 \%\right)$, or significant 


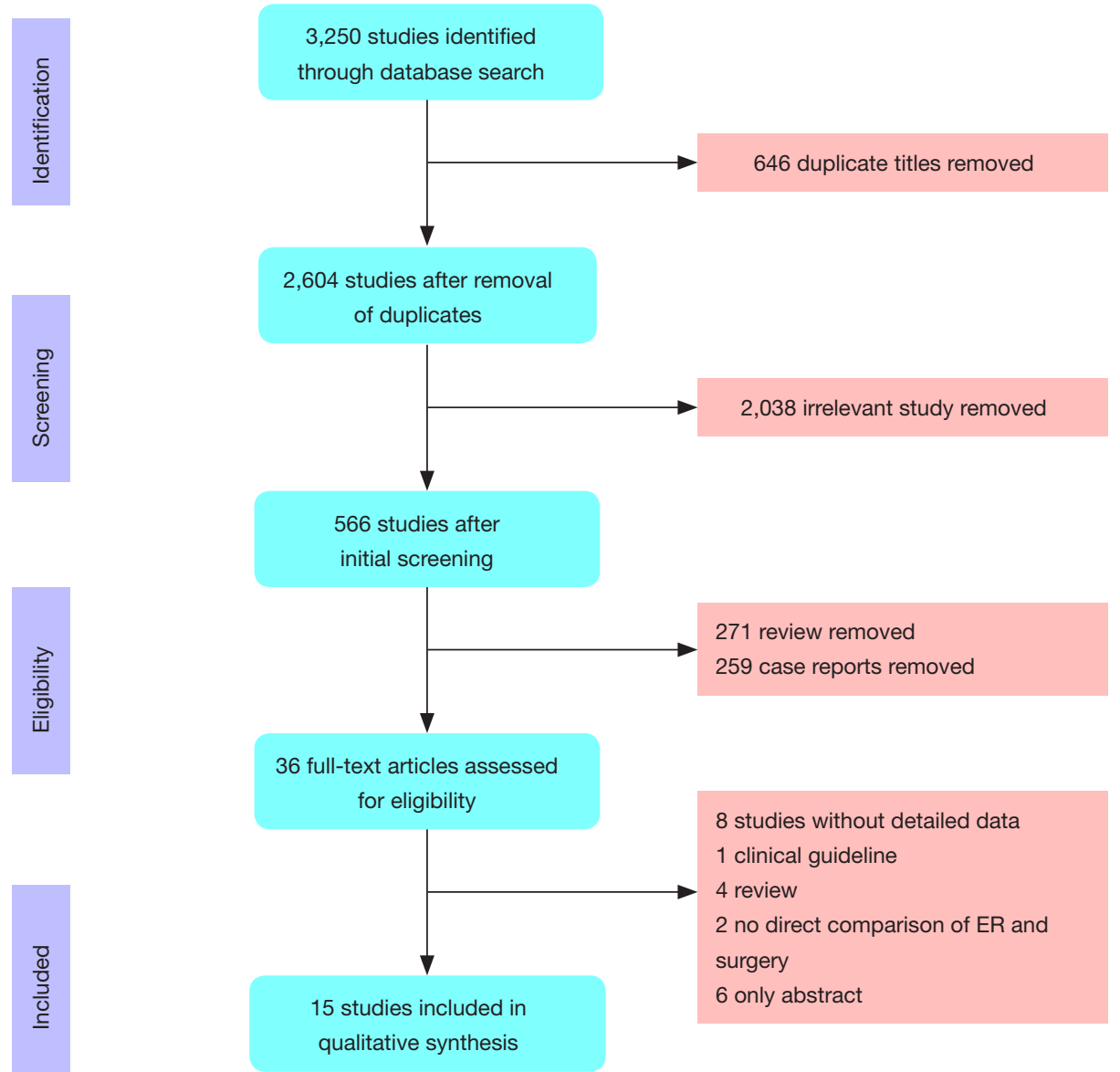

Figure 1 Schemata of the systematic review.

$\left(\mathrm{I}^{2}>50 \%\right)$. The $\chi^{2}$ test was used for the same purpose, with a statistical significance level of $\mathrm{P}<0.05$ indicating the presence of statistical heterogeneity. A sensitivity analysis was conducted to explore potential heterogeneity. Due to the expected clinical heterogeneity of the included studies, we chose to use only the random effects model before pooling the data.

\section{Results}

A total of 3,250 studies were identified by a search of the PubMed, EMBASE, and Cochrane Library databases. Of these, 2,684 irrelevant or duplicate studies were excluded, and an additional 530 studies were removed because they were reviews, abstracts, or case reports. A total of 36 records were eligible for full-text review, and 17 articles were excluded. Finally, 19 studies met the inclusion criteria and were thus included. The study flow chart is presented in Figure 1. There were 12 retrospective studies (9-20), three analyses using large national databases (21-23), and no randomized controlled trials (Table 1). Three studies were conducted in the United States, one in Germany, four in Korea, six in China, and one in Canada. This meta-analysis involved 4,731 patients, with 2,467 patients in the ER arm and 2,264 patients in the surgery arms.

Twelve studies reported major postprocedural adverse events. The incidence of major postprocedural adverse events ranged from $8-36 \%$ in the surgery group and $0-15 \%$ in the ER group. The meta-analysis indicated that ER was associated with fewer major adverse events than surgery [relative risk (RR), 0.46; 95\% confidence interval (CI), 0.33-0.64], with moderate heterogeneity $\left(\mathrm{I}^{2}=36 \%\right.$, $\mathrm{P}=0.10$ ) (Figure $2 A$ ). Procedure-related mortality was reported in five patients after ER and 30 patients after surgery. The meta-analysis showed that the ER group had a lower procedure-related mortality rate than the surgery group (RR, 0.27; 95\% CI, 0.10-0.73; $\mathrm{P}<0.01$ ), with no heterogeneity $\left(\mathrm{I}^{2}=15 \%, \mathrm{P}=0.31\right)$ (Figure $\left.2 B\right)$. Eight studies 


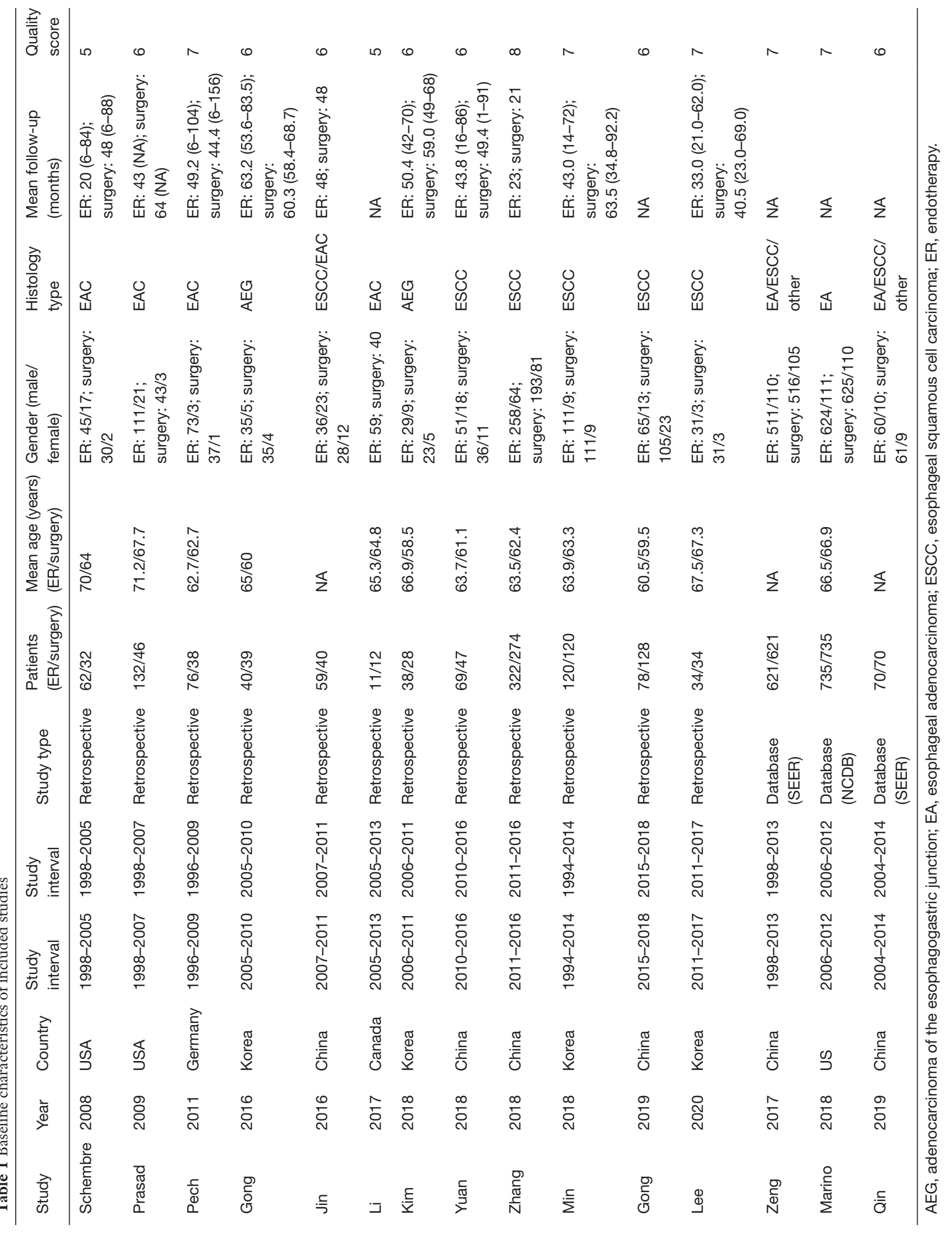


A Study

Schembre 2008

Prasad 2009

Pech 2011

Gong 2016

Jin 2016

Li 2017

Kim 2018

Yuan 2018

Zhang 2018

Min 2018

Gong 2019

Lee 2020

Random effects model

Heterogeneity: $I^{2}=36 \%, \tau^{2}=0.1066, p=0.10$

Test for overall effect: $z=-4.59(p<0.01)$

\section{Experimental Control Events Total Events Total}

$\begin{array}{rrrr}5 & 62 & 4 & 32 \\ 18 & 132 & 17 & 46 \\ 0 & 76 & 12 & 38 \\ 3 & 40 & 4 & 39 \\ 4 & 59 & 3 & 40 \\ 1 & 11 & 2 & 12 \\ 5 & 38 & 8 & 28 \\ 6 & 69 & 8 & 47 \\ 20 & 127 & 35 & 127 \\ 14 & 157 & 63 & 191 \\ 14 & 78 & 31 & 128 \\ 1 & 34 & 10 & 34 \\ & & & \end{array}$

B

Study

Schembre 2008

Prasad 2009

Pech 2011

Gong 2016

Jin 2016

Li 2017

Kim 2018

Yuan 2018

Zhang 2018

Min 2018

Lee 2020

Marino 2018

Random effects model

Heterogeneity: $I^{2}=15 \%, \tau^{2}=0.3101, p=0.31$

Test for overall effect: $z=-2.59(p<0.01)$

\section{Experimental Control Events Total Events Total}

\begin{tabular}{lr}
1 & 62 \\
0 & 132 \\
0 & 76 \\
0 & 40 \\
0 & 59 \\
0 & 11 \\
0 & 38 \\
0 & 69 \\
1 & 127 \\
0 & 157 \\
2 & 34 \\
1 & 735 \\
\multicolumn{3}{c}{1540} \\
$101, p=0.31$ \\
$p<0.01)$
\end{tabular}

Risk Ratio

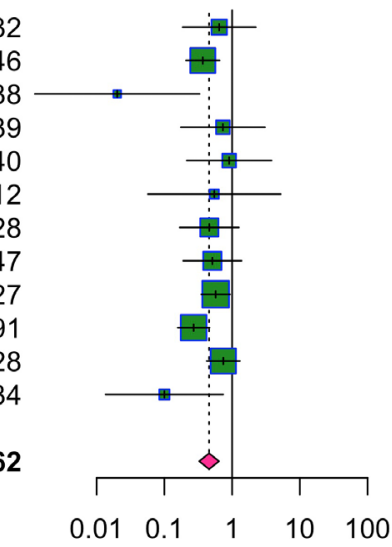

RR $\quad 95 \%-\mathrm{Cl}$ Weight

$0.65[0.19 ; 2.24] \quad 5.7 \%$

$0.37[0.21 ; 0.65] \quad 15.1 \%$

$0.02[0.00 ; 0.33] \quad 1.4 \%$

$0.73[0.17 ; 3.06] \quad 4.5 \%$

$0.90[0.21 ; 3.82] \quad 4.5 \%$

$0.55[0.06 ; 5.21] \quad 2.0 \%$

$0.46[0.17 ; 1.26] \quad 7.9 \%$

$0.51[0.19 ; 1.38] \quad 8.0 \%$

$0.57[0.35 ; 0.93] \quad 17.1 \%$

$0.27[0.16 ; 0.46] \quad 15.9 \%$

$0.74[0.42 ; 1.30] \quad 15.3 \%$

$0.10[0.01 ; 0.74] \quad 2.5 \%$

$0.46[0.33 ; 0.64] 100.0 \%$

Figure 2 Meta-analysis showed that endoscopic resection (ER) was associated with fewer major adverse events (RR, 0.46; 95\% CI, 0.330.64 ) and a lower procedure-related mortality rate (RR, 0.27; 95\% CI, 0.10-0.73) than the surgery group. (A) The comparison of major postprocedural adverse events between endoscopic resection and surgery. (B) The comparison of procedure-related mortality between endoscopic resection and surgery. RR, relative risk; CI, confidence interval.

reported postprocedural stricture events. The metaanalysis revealed that the frequency of postprocedural stricture events did not significantly differ between ER and surgery (RR, 0.89; 95\% CI, 0.53-1.49), with significant heterogeneity $\left(\mathrm{I}^{2}=53 \%, \mathrm{P}=0.04\right)$ (Figure 3$)$. The sensitivity analysis was performed to explore the heterogeneity. After deletion, the pooled result did not deviate beyond the $95 \%$ CI of the whole estimate (Figure S1).

The $\mathrm{R} 0$ resection rate is a vital parameter for evaluating the efficacy of treatment and recurrence. In this meta- analysis, nine studies reported the $\mathrm{R} 0$ resection rate after treatment. The R0 resection rate was $97-100 \%$ in patients who underwent esophagectomy and $73-100 \%$ in patients who underwent ER treatment. The pooled outcomes indicated that the R0 resection rate was lower in the ER group than in the surgery group (RR, 0.92; $95 \%$ CI, 0.86-0.98), with significant heterogeneity $\left(\mathrm{I}^{2}=91 \%\right.$, $\mathrm{P}<0.01$ ) (Figure $4 A$ ). According to the sensitivity analysis, although the data from the study by Gong et al. were seen as a potential source of heterogeneity, the combined RR 


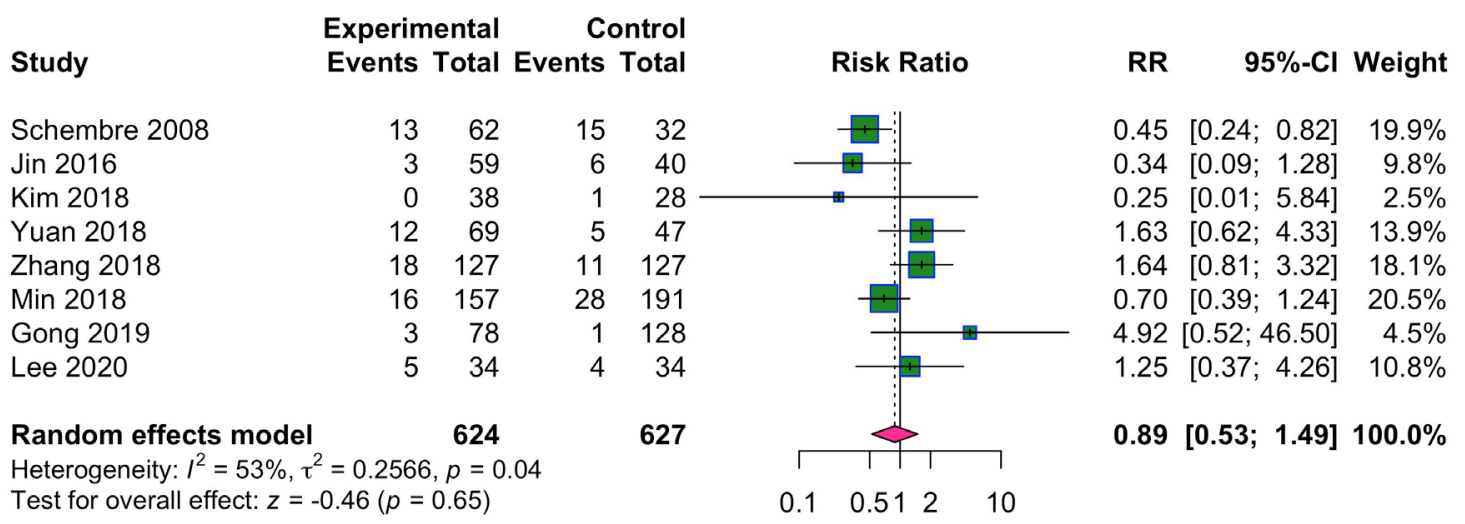

Figure 3 The comparison of postprocedural stricture events between endoscopic resection and surgery. RR, relative risk; CI, confidence interval.

A Study

Prasad 2009

Pech 2011

Gong 2016

Jin 2016

Kim 2018

Yuan 2018

Zhang 2018

Gong 2019

Lee 2020

Random effects model

Heterogeneity: $I^{2}=91 \%, \tau^{2}=0.0088, p<0.01$

Test for overall effect: $z=-2.54(p=0.01)$

$64 \quad 69$

$118 \quad 127$

$57 \quad 78$

$25 \quad 34$

653

\section{Experimental Control Events Total Events Total}

$\begin{array}{llll}124 & 132 & 46 & 46\end{array}$

$\begin{array}{llll}75 & 76 & 38 & 38\end{array}$

$\begin{array}{llll}35 & 40 & 39 & 39\end{array}$

$\begin{array}{llll}56 & 59 & 39 & 40\end{array}$

$\begin{array}{llll}38 & 38 & 28 & 28\end{array}$

0.01

$\begin{array}{ll}47 & 47\end{array}$

$124 \quad 127$

128

3434

527

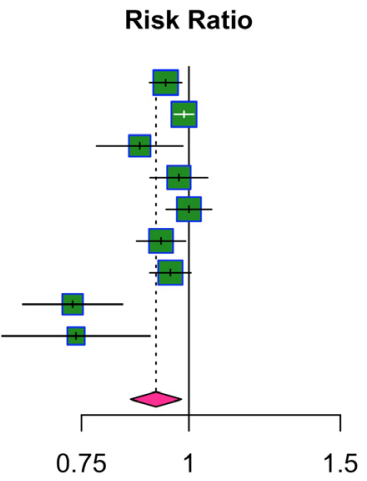

RR $\quad 95 \%-\mathrm{Cl}$ Weight

$0.94[0.90 ; 0.98] 12.9 \%$

$0.99[0.96 ; 1.01] 13.4 \%$

$0.88[0.78 ; 0.98] \quad 9.8 \%$

0.97 [0.90; 1.05] $11.6 \%$

$1.00[0.94 ; 1.06] 12.3 \%$

$0.93[0.87 ; 0.99] \quad 12.1 \%$

$0.95[0.90 ; 1.01] \quad 12.5 \%$

$0.73[0.64 ; 0.84] \quad 8.9 \%$

$0.74[0.61 ; 0.90] \quad 6.3 \%$

$0.92[0.86 ; 0.98] 100.0 \%$

B

Study

Schembre 2008

Prasad 2009

Pech 2011

Gong 2016

Jin 2016

Li 2017

Kim 2018

Yuan 2018

Zhang 2018

Lee 2020

\section{Experimental Control} Events Total Events Total

Random effects model

Heterogeneity: $I^{2}=0 \%, \tau^{2}=0, p=0.9$

Test for overall effect: $z=1.93(p=0.05)$

$\begin{array}{rrrr}4 & 62 & 0 & 32 \\ 16 & 132 & 1 & 46 \\ 1 & 76 & 0 & 38 \\ 0 & 40 & 0 & 39 \\ 6 & 59 & 3 & 40 \\ 1 & 11 & 1 & 12 \\ 2 & 38 & 0 & 28 \\ 6 & 69 & 2 & 47 \\ 12 & 127 & 9 & 127 \\ 3 & 34 & 2 & 34 \\ & & & \\ & 648 & & 443\end{array}$

443

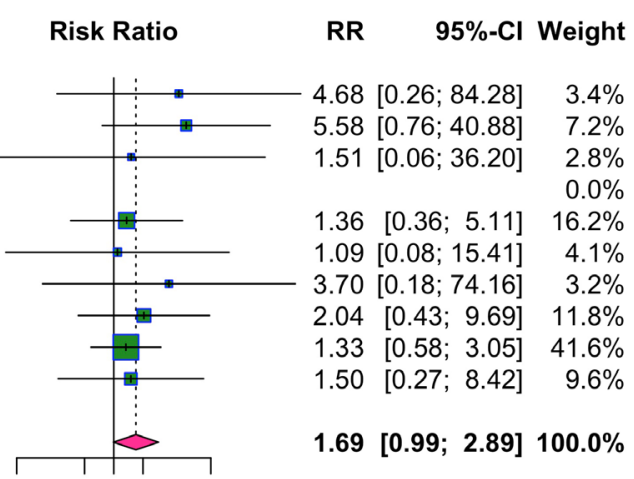

$\begin{array}{llll}0.1 & 0.512 & 10\end{array}$

Figure 4 Meta-analysis showed that endoscopic resection (ER) was associated with a lower R0 resection rate (RR, 0.92; 95\% CI, 0.86-0.98) and a higher tumor recurrence rate (RR, 1.69; 95\% CI, 0.99-2.89) than the surgery group. (A) The comparison of R0 resection rate between endoscopic resection and surgery. (B) The comparison of recurrence rate between endoscopic resection and surgery. RR, relative risk; CI, confidence interval. 


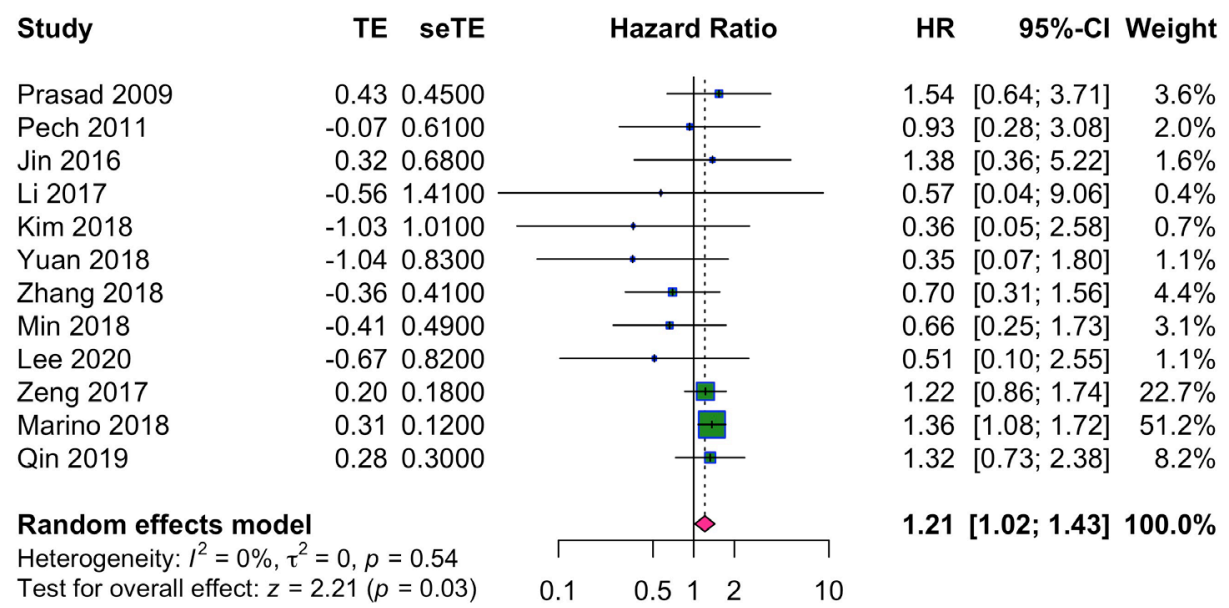

Figure 5 The comparison of overall survival between endoscopic resection and surgery. HR, hazard ratio; CI, confidence interval.

was very close to the original $\mathrm{RR}$ and the pooled result did not deviate beyond the $95 \% \mathrm{CI}$ of the whole estimate (Figure S2). Ten studies reported tumor recurrence during follow-up. The meta-analysis demonstrated that the ER group had a higher tumor recurrence rate than the surgery group (RR, 1.69; 95\% CI, 0.99-2.89), with no heterogeneity $\left(\mathrm{I}^{2}=0 \%, \mathrm{P}=0.94\right)$ (Figure 4B).

Twelve studies compared the OS between ER and surgery. The pooled result indicated poorer OS in the ER group than in the surgery group (HR, 1.21; 95\% CI, 1.02$1.43)$, with no heterogeneity $\left(\mathrm{I}^{2}=0 \%, \mathrm{P}=0.54\right)$ (Figure 5).

\section{Discussion}

In this meta-analysis, we aimed to compare the efficacy and safety of ER and surgery for EEC and found that the OS rate was better among patients who underwent surgery, and the recurrence rate was higher among those who underwent ER. More major adverse events were recorded and the procedure-related mortality was higher among those who underwent surgery, but the R0 resection rate was lower among those who underwent ER. However, the rate of postprocedural stricture events did not significantly differ between the two treatments.

Based on favorable safety and oncologic outcomes in suitable indications, ER therapy is increasing in popularity worldwide for T1 esophageal cancer (24). In contrast to the high incidence of major adverse events in esophagectomy, ER is associated with few adverse events and almost never results in perioperative death. In addition, the major adverse events of ER such as bleeding and stenosis also can be successfully treated with endoscopic procedures. Our results indicated that ER was associated with fewer major adverse events and a lower procedure-related mortality rate. Esophageal stricture as a major complication that manifests as varying degrees of dysphagia, which greatly increases medical costs and reduces quality of life. In our research, the pooled results indicated that the postoperative stricture rate was comparable between the ER group and the surgery group $(11.2 \%$ vs. $11.3 \%, \mathrm{P}=0.65)$.

Endoscopic ultrasound (EUS) is the most commonly used clinical staging modality but has an overall accuracy of only $\sim 65 \%$ (25), which may affect treatment choice or outcomes. Even in clinical T1a patients, $20.2 \%$ of patients with deep invasion after esophagectomy and $8.7 \%$ of patients with occult lymph node metastasis were missed by EUS (24). Further, once a tumor breaches the muscularis mucosae, the incidence of lymphatic involvement exceeds $30 \%$, and it is generally accepted that ER therapy no longer provides curative ability (26). In addition, our study found that the recurrence rate was significantly higher in the ER group than in the surgery group $(\mathrm{P}=0.05)$. Therefore, accurate clinical staging is crucial to treatment selection and the overall outcome. For patients treated with ER, postoperative monitoring is critical for detecting local tumor recurrence, which can be treated with esophagectomy. It is worth noting that, among the included studies, Jin et al. (13) compared the ER therapy and minimally invasive esophagectomy (MIE) and found no significant differences in the incidence of major complications between the two 
treatment groups $(\mathrm{P}=1.0)$. Gong et al. (19) compared 128 patients who underwent MIE with 78 patients treated with ESD, of which $77 \%$ were T1a in the ESD group and 34\% T1a in the MIE group. Again, there was no difference in overall postoperative complication rates $(24.22 \% v s$. $23.08 \% ; \mathrm{P}=1.000)$. According to these findings, under the premise of the completeness of resection and radical lymphadenectomy, MIE would not significantly increase the main complications. Therefore, ER therapy is a less invasive treatment for T1a patients, whereas MIE therapy may be more suitable for $\mathrm{T} 1 \mathrm{~b}$ patients or patients at greater risk of lymph node metastases.

Some studies comparing the long-term survival rate associated with the two procedures used the Surveillance Epidemiology and End Results (SEER) registry or the National Cancer Database (NCDB). Most studies reported no difference in OS or cancer-specific survival between the two treatment groups. However, Marino et al. (22) used the NCDB after propensity matching and found that ER therapy positively affected survival during the first 90 days (HR, 0.15; $\mathrm{P}=0.003$ ), whereas esophagectomy was associated with better survival after 90 days (HR, 1.34; $\mathrm{P}=0.02$ ). Similarly, Zeng et al. (21) used the SEER registry and also found poorer OS $(\mathrm{HR}, 1.690 ; \mathrm{P}<0.001)$ in the ER group than in the esophagectomy group. Nevertheless, all single-center retrospective studies included in this metaanalysis reported no difference in the long-term survival rate. Only one study by Prasad et al. (10) found that cancerfree survival was lower in the ER group than in the surgery group $(80 \%$ vs. $97 \%, \mathrm{P}=0.01)$. In our study, the pooled results showed that the OS was poorer in the ER group than in the surgery group (HR, 1.21; 95\% CI, 1.02-1.43; $\mathrm{P}=0.03)$. Most of the included studies were single-center retrospective studies, and the number of included cases was relatively small. Some studies used public databases that did not include propensity-score matching, which may have resulted in significant selection bias. However, our research revealed a higher survival rate in the surgery group than the ER group, which may have been due to the higher risk of occult positive lymph nodes in T1b disease and the improved locoregional control with esophagectomy.

Several limitations of this meta-analysis need to be considered. First, all of the included studies were retrospective and uncontrolled. The relatively small number of cases in some studies may have reduced the reliability of the findings. Second, the large database studies have several common shortcomings. One of the more important limitations is the inability to verify important data points, such as pathologic diagnosis and comorbidities. In addition, the databases do not contain records of local therapies used before surgery, including whether or not ER was performed. Third, some of the HRs were extracted by calculations, which inevitably introduces bias. Finally, different types of esophageal tumors, ER procedures, and surgical approaches may have resulted in biased results, but the included studies were limited and subgroup analyses could not be performed.

In conclusion, ER for EEC is associated with fewer major adverse events and lower procedure-related mortality rate than surgery, but this therapy does not address potential lymphatic spread. On the contrary, esophagectomy with lymph node dissection has a higher $\mathrm{R} 0$ resection rate and lower tumor recurrence rate, which may result in improved survival. Thus, ER cannot completely replace radical surgery for EEC. Long-term follow-up is critical for patients undergoing ER.

\section{Acknowledgments}

Funding: None.

\section{Footnote}

Reporting Checklist: The authors have completed the PRISMA reporting checklist. Available at https://dx.doi. org/10.21037/tcr-21-182

Peer Review File: Available at https://dx.doi.org/10.21037/ tcr-21-182

Conflicts of Interest: All authors have completed the ICMJE uniform disclosure form (available at https://dx.doi. org/10.21037/tcr-21-182). The authors have no conflicts of interest to declare.

Ethical Statement: The authors are accountable for all aspects of the work in ensuring that questions related to the accuracy or integrity of any part of the work are appropriately investigated and resolved.

Open Access Statement: This is an Open Access article distributed in accordance with the Creative Commons Attribution-NonCommercial-NoDerivs 4.0 International License (CC BY-NC-ND 4.0), which permits the noncommercial replication and distribution of the article with the strict proviso that no changes or edits are made and the original work is properly cited (including links to both the 
formal publication through the relevant DOI and the license). See: https://creativecommons.org/licenses/by-nc-nd/4.0/.

\section{References}

1. Rice TW, Rusch VW, Ishwaran H, et al. Cancer of the esophagus and esophagogastric junction: datadriven staging for the seventh edition of the American Joint Committee on Cancer/International Union Against Cancer Cancer Staging Manuals. Cancer 2010;116:3763-73.

2. Wijnhoven BP, Tran KT, Esterman A, et al. An evaluation of prognostic factors and tumor staging of resected carcinoma of the esophagus. Ann Surg 2007;245:717-25.

3. Finks JF, Osborne NH, Birkmeyer JD. Trends in hospital volume and operative mortality for high-risk surgery. $\mathrm{N}$ Engl J Med 2011;364:2128-37.

4. Scarpa M, Valente S, Alfieri R, et al. Systematic review of health-related quality of life after esophagectomy for esophageal cancer. World J Gastroenterol 2011;17:4660-74.

5. Bennett C, Vakil N, Bergman J, et al. Consensus statements for management of Barrett's dysplasia and early-stage esophageal adenocarcinoma, based on a Delphi process. Gastroenterology 2012;143:336-46.

6. Gertler R, Stein H, Schuster T, et al. Prevalence and topography of lymph node metastases in early esophageal and gastric cancer. Ann Surg 2014;259:96-101.

7. Stang A. Critical evaluation of the Newcastle-Ottawa scale for the assessment of the quality of nonrandomized studies in meta-analyses. Eur J Epidemiol 2010;25:603-5.

8. Tierney JF, Stewart LA, Ghersi D, et al. Practical methods for incorporating summary time-to-event data into metaanalysis. Trials 2007;8:16.

9. Schembre DB, Huang JL, Lin OS, et al. Treatment of Barrett's esophagus with early neoplasia: a comparison of endoscopic therapy and esophagectomy. Gastrointest Endosc 2008;67:595-601.

10. Prasad GA, Wu TT, Wigle DA, et al. Endoscopic and surgical treatment of mucosal (T1a) esophageal adenocarcinoma in Barrett's esophagus. Gastroenterology 2009;137:815-23.

11. Pech O, Bollschweiler E, Manner H, et al. Comparison between endoscopic and surgical resection of mucosal esophageal adenocarcinoma in Barrett's esophagus at two high-volume centers. Ann Surg 2011;254:67-72.

12. Gong EJ, Kim DH, Ahn JY, et al. Comparison of longterm outcomes of endoscopic submucosal dissection and surgery for esophagogastric junction adenocarcinoma.
Gastric Cancer 2017;20:84-91.

13. Jin XF, Gai W, Chai TH, et al. Comparison of Endoscopic Resection and Minimally Invasive Esophagectomy in Patients with Early Esophageal Cancer. J Clin Gastroenterol 2017;51:223-7.

14. Li C, Yamashita D, Hawel J, et al. Endoscopic mucosal resection versus esophagectomy for intramucosal adenocarcinoma in the setting of barrett's esophagus. Surg Endosc 2017;31:4211-6.

15. Kim HJ, Chung H, Shin SK, et al. Comparison of long-term clinical outcomes between endoscopic and surgical resection for early-stage adenocarcinoma of the esophagogastric junction. Surg Endosc 2018;32:3540-7.

16. Yuan B, Liu L, Huang H, et al. Comparison of the shortterm and long-term outcomes of surgical treatment versus endoscopic treatment for early esophageal squamous cell neoplasia larger than $2 \mathrm{~cm}$ : a retrospective study. Surg Endosc 2019;33:2304-12.

17. Zhang Y, Ding H, Chen T, et al. Outcomes of Endoscopic Submucosal Dissection vs Esophagectomy for T1 Esophageal Squamous Cell Carcinoma in a Real-World Cohort. Clin Gastroenterol Hepatol 2019;17:73-81. e3. Erratum in: Clin Gastroenterol Hepatol. 2020 Mar;18(3):758. doi: 10.1016/j.cgh.2020.01.012.

18. Min YW, Lee H, Song BG, et al. Comparison of endoscopic submucosal dissection and surgery for superficial esophageal squamous cell carcinoma: a propensity score-matched analysis. Gastrointest Endosc 2018;88:624-33.

19. Gong L, Yue J, Duan X, et al. Comparison of the therapeutic effects of endoscopic submucosal dissection and minimally invasive esophagectomy for $\mathrm{T} 1$ stage esophageal carcinoma. Thorac Cancer 2019;10:2161-7.

20. Lee HD, Chung H, Kwak Y, et al. Endoscopic Submucosal Dissection Versus Surgery for Superficial Esophageal Squamous Cell Carcinoma: A Propensity ScoreMatched Survival Analysis. Clin Transl Gastroenterol 2020;11:e00193.

21. Zeng Y, Liang W, Liu J, et al. Endoscopic Treatment Versus Esophagectomy for Early-Stage Esophageal Cancer: a Population-Based Study Using Propensity Score Matching. J Gastrointest Surg 2017;21:1977-83.

22. Marino KA, Sullivan JL, Weksler B. Esophagectomy versus endoscopic resection for patients with early-stage esophageal adenocarcinoma: A National Cancer Database propensity-matched study. J Thorac Cardiovasc Surg 2018;155:2211-2218.e1.

23. Qin J, Peng Y, Chen W, et al. Comparative study of 
esophagectomy, endoscopic therapy, and radiotherapy for cT1N0M0 esophageal cancer in elderly patients: A SEER database analysis. Thorac Cancer 2019;10:1511-20.

24. Semenkovich TR, Hudson JL, Subramanian M, et al. Trends in Treatment of T1N0 Esophageal Cancer. Ann Surg 2019;270:434-43.

25. Young PE, Gentry AB, Acosta RD, et al. Endoscopic

Cite this article as: Zheng H, Kang N, Huang Y, Zhao Y, Zhang R. Endoscopic resection versus esophagectomy for early esophageal cancer: a meta-analysis. Transl Cancer Res 2021;10(6):2653-2662. doi: 10.21037/tcr-21-182 ultrasound does not accurately stage early adenocarcinoma or high-grade dysplasia of the esophagus. Clin Gastroenterol Hepatol 2010;8:1037-41.

26. Gan S, Watson DI. New endoscopic and surgical treatment options for early esophageal adenocarcinoma. J Gastroenterol Hepatol 2010;25:1478-84. 


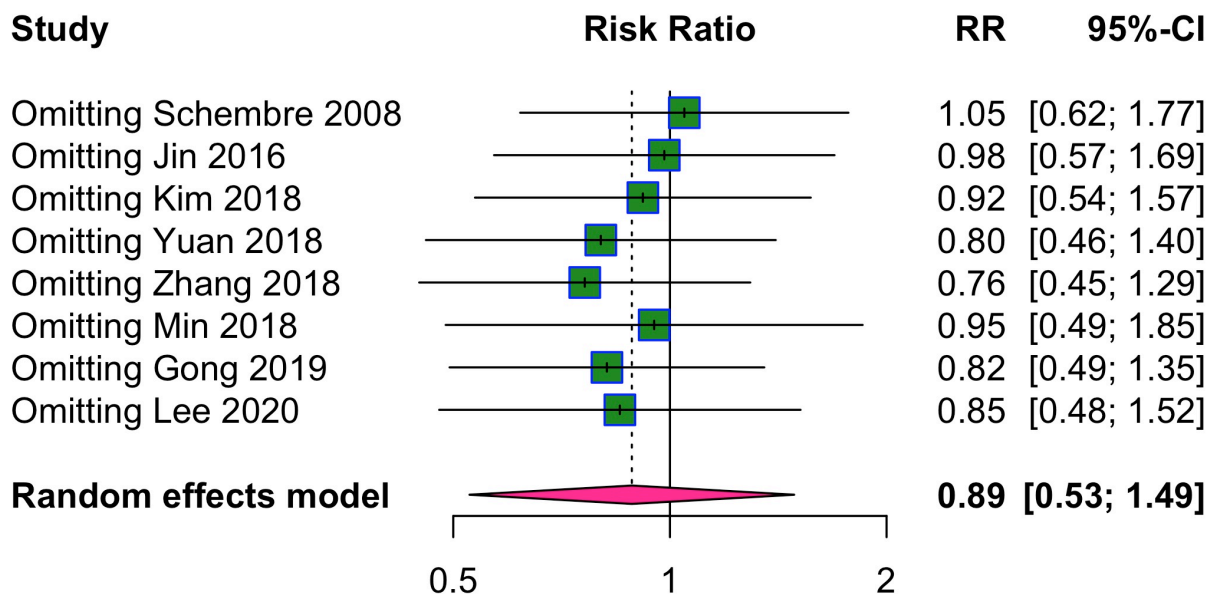

Figure S1 Sensitivity analysis of postprocedural stricture events. RR, relative risk; CI, confidence interval.

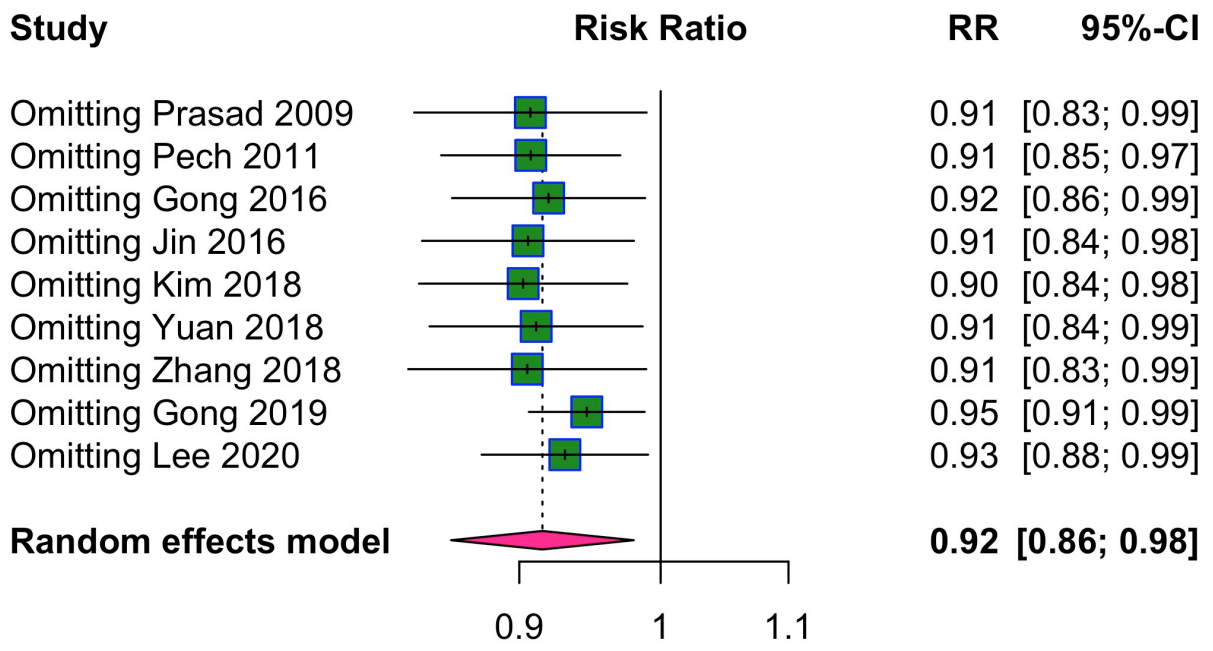

Figure S2 Sensitivity analysis of R0 resection rate. RR, relative risk; CI, confidence interval. 